\title{
Scheduled Notification
}

National Cancer Institute

\section{Source}

National Cancer Institute. Scheduled Notification. NCI Thesaurus. Code C93414.

The communication of a message to a recipient that is anticipated to occur at some time in the future and has been assigned a time or date when that activity is to be performed. 